\title{
BODIPY-conjugated chitosan nanoparticles as a fluorescent probe
}

\section{Gizem Bor, Muhammed Üçüncü, Mustafa Emrullahoğlu, Aysel Tomak \& Gülşah Şanlı-Mohamed}

To cite this article: Gizem Bor, Muhammed Üçüncü, Mustafa Emrullahoğlu, Aysel Tomak \& Gülșah Șanlı-Mohamed (2016): BODIPY-conjugated chitosan nanoparticles as a fluorescent probe, Drug and Chemical Toxicology, DOI: 10.1080/01480545.2016.1238481

To link to this article: http://dx.doi.org/10.1080/01480545.2016.1238481

\section{曲 Published online: 21 Nov 2016.}

\section{Submit your article to this journal $\widetilde{ }$}

Llll Article views: 70

Q View related articles $\longleftarrow$

View Crossmark data ¿ 


\title{
BODIPY-conjugated chitosan nanoparticles as a fluorescent probe
}

\author{
Gizem Bor ${ }^{1}$, Muhammed Üçüncü ${ }^{2}$, Mustafa Emrullahoğlu ${ }^{2}$, Aysel Tomak ${ }^{3}$, and Gülşah Şanlı-Mohamed ${ }^{2}$ \\ ${ }^{1}$ Biotechnology and Bioengineering Department, ${ }^{2}$ Chemistry Department and ${ }^{3}$ Materials Science and Engineering Department, Izmir Institute of \\ Technology, Izmir, Turkey
}

\begin{abstract}
Recently, development of fluorescent nanoparticle-based probes for various bioimaging applications has attracted great attention. This work aims to develop a new type fluorescent nanoparticle conjugate and evaluate its cytotoxic effects on A549 and BEAS 2B cell lines. Throughout the study, ionically crosslinked chitosan nanoparticles (CNs) were conjugated with carboxylated 4,4-difluoro-4-bora-3a,4a-diaza-s-indacene (BODIPY-COOH). The results of conjugates (BODIPY-CNs) were investigated with regard to their physic-chemical, optical, cytotoxic properties and cellular internalization. The morphology of BODIPY-CNs was found to be spherical in shape and quite uniform having average diameter of $70.25 \pm 11.99 \mathrm{~nm}$. Cytotoxicty studies indicated that although BODIPY-COOH itself was quite toxic on both A549- and BEAS 2B-treated cells, CNs increased the cell viability of both cell lines via conjugation to BODIPY$\mathrm{COOH}$ fluorescent molecule up to $67 \%$ for A549 and $74 \%$ for BEAS $2 \mathrm{~B}$ cells. These results may suggest a possible utilization of the new fluorescent nanoparticle-based probe for bioimaging in biology and medicine.
\end{abstract}

\section{Keywords}

Chitosan, nanoparticles, BODIPY, cytotoxicity, A549 cells, BEAS 2B cells

\section{History}

Received 22 March 2016

Revised 11 August 2016

Accepted 9 September 2016

\section{Introduction}

In recent years, bioimaging applications with fluorescent probes have attracted important attention in biology and medicine (Chekina et al., 2011; Corr et al., 2008; Terai \& Nagano, 2008; Yuan et al., 2013). Especially, bioimaging applications with fluorescent molecules on cancer cells are highly promising with the possibility of real-time visual detection of an analyte both in solution and living organism (Chan et al., 2012).

Organic fluorescent dyes have been greatly applied in the synthesis of fluorescent probes for molecular imaging and biomedical diagnosis (Kobayashi et al., 2010). Borondipyrromethene (BODIPY), a type of organic fluorescent molecules, has remarkable photophysical properties such as long excitation/emission wavelengths, high molar absorption coefficients and fluorescence quantum yield (Boens et al., 2012; Ulrich et al., 2008; Loudet \& Burgess, 2007). Thus, the BODIPY fluorophore can be a good candidate to be utilized as the organic fluorescent molecules for bioimaging applications (Bernhard et al., 2010). On the other hand, organic dyes are not very stable due to photobleaching problem, low-signal intensities and wide fluorescent spectrum limitations. It has lately been reported that, conjugation of organic dyes with polymer-based nanoparticles has increased their stability

Address for correspondence: Assoc. Prof. Dr. Gülşah Şanl1-Mohamed, Izmir Institute of Technology, Science Faculty, Department of Chemistry, Urla, Izmir, Turkey. Tel: +90 2327507618. Fax: +90 2327507509. E-mail: gulsahsanli@iyte.edu.tr; gulsahsanli@ hotmail.com compared to common single organic fluorophores (Nune et al., 2009).

Chitosan nanoparticles have attracted a remarkable attention lately. As a potential biomedical material, chitosan, a poly-N-acetyl glucosamine, is the $\mathrm{N}$-deacetylated product of chitin (major component of crustacean shells), which is the second most abundant biopolymer after cellulose (Kumar et al., 2004). Chitosan, a type of cationic polysaccharide, has many significant biological and chemical properties due to a unique set of characteristics that include biocompatibility, biodegradability, nontoxicity, physiological inertness and antibacterial properties (Rinaudo, 2006). Therefore, chitosan can be used for nano-sized materials in biomedicine for variety applications (Jayakumar et al., 2010). Chitosan as nanoparticles are reported to be a suitable polymer-based material for loading organic fluorophores because of their high labeling affinity to various biomolecules (Zhao \& Wu, 2006).

Although, many studies have been conducted for the development of chitosan nanoparticles with variety of fluorescent probes for the purpose of bioimaging applications, studies of BODIPY conjugated chitosan nanoparticles have not been reported. The objective of this study is to obtain BODIPY-conjugated chitosan nanoparticles as fluorescent probe and evaluate their cytotoxic effect on A549 and BEAS 2B cell lines. In the development of a functional fluorescent nano-size probe, initially, CNs were prepared based on ionic gelation method and BODIPY-COOH fluorescent dye was synthesized as a type of organic fluorescent molecule. CNs were then conjugated with BODIPY-COOH and the 
conjugation was confirmed by visualizing the newly formed compound under UV light and confocal microscope. The physicochemical properties of the CNs and BODIPY-COOH conjugated $\mathrm{CNs}$ were determined by several methods including Scanning Electron Microscopy (SEM), Fourier Transform Infrared Spectroscopy (FT-IR), Dynamic Light Scattering (DLS), Atomic Force Microscopy (AFM). Cytotoxic effects of CNs, BODIPY-COOH and BODIPYCNs were evaluated comparing cell viability of A549 and BEAS 2B cell lines. According to our results, a newly formed fluorescent nanoparticle-based probe could be a good candidate for potential bioimaging applications in biology and medicine.

\section{Materials and methods}

\section{Materials}

Chitosan (190-310 kDa and degree of acetylation 75-85\%), tripolyphosphate sodium (TPP), 3-[4,5-dimethylthiazol-2-yl]2,5-diphenylterazolium bromide (MTT) and 4',6-diamidino2-phenylindole (DAPI) were obtained from Sigma-Aldrich (St. Louis, MO). All the other chemicals were obtained from local chemical suppliers.

\section{Synthesis of BODIPY-COOH}

Carboxylated 4,4-difluoro-4-bora-3a,4a-diaza-s-indacene (BODIPY-COOH) was synthesized by the method of Brizet et al. (2013) with some modification. In the procedure, to an anhydrous dichloromethane (DCM) $(50 \mathrm{~mL})$ of 2,4-dimethylethylpyrrole (DMAP) $(739.2 \mathrm{mg}, 6.0 \mathrm{mmol}), 4$-carboxy-benzaldehyde $(450.4 \mathrm{mg}, 3.0 \mathrm{mmol})$ and catalytic amount of trifluoroacetic acid (2-3 drops) were added. The reaction mixture was stirred at room temperature for $24 \mathrm{~h}$. A solution of p-chloranil $(1.475 \mathrm{~g}, 6.0 \mathrm{mmol})$ in dichloromethane $(10 \mathrm{~mL})$ was added and the mixture was stirred for $1 \mathrm{~h}$. Triethylamine $(6 \mathrm{~mL}, 45 \mathrm{mmol})$ and $\mathrm{BF}_{3} \cdot \mathrm{Et}_{2} \mathrm{O}(6 \mathrm{~mL}$, $47.4 \mathrm{mmol}$ ) were added successively. The purple solution was stirred $4 \mathrm{~h}$. The reaction mixture was extracted with dichloromethane $(3 \times 100 \mathrm{~mL})$, dried over sodium sulfate and concentrated under vacuum. The residue was purified by silica gel column chromatography (Hex:EtAc 4:1). The resulting solid was further purified by recrystallization (chloroform-hexane system) to give title product as a red solid (Yield: $35 \%, \mathrm{~m}=445 \mathrm{mg}) .1 \mathrm{H} \quad \mathrm{NMR} \quad(400 \mathrm{MHz}$, $\left.\mathrm{CDCl}_{3}\right): \delta 8.26(\mathrm{~d}, J=8.4 \mathrm{~Hz}, 2 \mathrm{H}), 7.46(\mathrm{~d}, J=7.4 \mathrm{~Hz}, 2 \mathrm{H})$, $2.54(\mathrm{~s}, 6 \mathrm{H}), 2.31(\mathrm{q}, J=7.6 \mathrm{~Hz}, 4 \mathrm{H}), 1.28(\mathrm{~s}, 6 \mathrm{H}), 0.99$ $(\mathrm{t}, J=7.6 \mathrm{~Hz}, 6 \mathrm{H}) .13 \mathrm{C} \mathrm{NMR}(100 \mathrm{MHz}, \mathrm{CDCl} 3): \delta 171.0$, 154.4, 141.7, 138.4, 138.0, 133.1, 130.8, 130.2, 129.7, 128.9, 17.1, 14.6, 12.6, 11.8 .

\section{Preparation of chitosan nanoparticles (CNs)}

Chitosan nanoparticles (CNs) were synthesized by following the Calvo's ionic gelation procedure (Calvo et al., 1997). According to this procedure, tripolyphosphate (TPP) was used as a crosslinker with chitosan. Chitosan was dissolved in 1\% (w/ v) acetic acid solution. Then, the $\mathrm{pH}$ of the solution was adjusted to 5.0 with $3.0 \mathrm{M} \mathrm{NaOH}$ solution. The aqueous solution of TPP was obtained as $0.1 \%$ in acetic acid solution. Chitosan nanoparticles started to form spontaneously upon addition of
TPP into the solution of chitosan under magnetic stirring drop wise at room temperature. After one hour of mixing chitosan with TPP, the solution was centrifuged $(13500 \mathrm{rpm} / 30 \mathrm{~min}$ ) and the pellet was freeze-dried by lyophilization.

\section{Preparation and characterization of BODIPY-CNs}

For preparation of BODIPY-CNs, freeze-dried chitosan nanoparticles were added to $5 \mathrm{ml}$ of DMSO under constant magnetic stirrer. To this solution, BODIPY-COOH solution [ $5 \mathrm{mg}$ in $1 \mathrm{~mL}$ DMSO and preactivated with $0.5 \mathrm{mg}$ EDC (1-Ethyl-3-(3-dimethylaminopropyl) carbodiimide reagent in $1 \mathrm{~mL}$ of $\mathrm{pH} 5.0 \mathrm{NaP}$ buffer] was added drop wise. The reaction between $\mathrm{CNs}$ and $\mathrm{BODIPY-COOH}$ was allowed to continue for $24 \mathrm{~h}$ on the magnetic stirrer at room temperature. BODIPY-CNs were then centrifuged at $13500 \mathrm{rpm}$ for $30 \mathrm{~min}$ and washed with DMSO several times until the free BODIPY-COOH was not left in the supernatant. The BODIPY-COOH concentration was measured by UV-visible Spectrophotometry (Shimadzu-UV 2540, Kyoto, Japan) calibrated with standard solutions containing $0.005-0.5 \mu \mathrm{g} /$ $\mathrm{mL}$ of BODIPY-COOH dissolved in DMSO. Binding efficiency (w/w \%) of the conjugate was calculated at the maximum wavelength of $510 \mathrm{~nm}$.

The measurement of particle size (Z-average) of nanoparticles were performed on a Zetasizer Nano-ZS (Malvern Zetasizer, 3000 HSA, Worcestershire, UK) on the basis of dynamic light scattering (DLS) techniques. The measurements were performed in triplicates at a temperature of $25^{\circ} \mathrm{C}$ and the samples were diluted with distilled water and measured at scattering angle of $173^{\circ}$. Changes in functional groups of chitosan nanoparticle after being conjugated with BODIPY-COOH were characterized by FT-IR (Perkin Elmer Spectrum 100, Waltham, MA) according to the potassium bromide pellet method on a Tensor-27 (Bruker, Billerica, MA) in the range of $800-4000 \mathrm{~cm}^{-1}$. Visualization of the morphology of the particles was performed by Atomic Force Microscope on tapping mode (Digital Instruments Nanoscope, Santa Barbara, CA) and Scanning Electron Microscope (SEM, FEI QUANTA 250 FEG, Hillsboro, OR).

The optical property of BODIPY-CNPs was determined by recording the fluorescence emission spectra using excitation at the range of $400-500 \mathrm{~nm}$ with a fluorescence spectrophotometer (Perkin Elmer).

\section{Cell culture}

BEAS 2B (human bronchial epithelial cell line) and A549 (adenocarcinoma human alveolar basal epithelial cells) cells lines were provided by the Biotechnology and Bioengineering Research and Application Center, IZTECH. The cells were grown in Roswell Park Memorial Institute-1640 (RPMI-1640) growth medium containing 10\% fetal bovine serum (FBS) and $1 \%$ gentamicin sulfate in a humidified atmosphere containing $5 \% \mathrm{CO}_{2}$ at $37^{\circ} \mathrm{C}$. Typically, cells were passaged through trypsinization and in growth medium.

\section{In vitro cellular uptake of BODIPY-CNs}

For the visualization of cells treated with BODIPY-CNs and BODIPY-COOH, confocal microscopy (Olympus-CKX41, 
Tokyo, Japan) was used for A549 cell lines. The cells of passage 14 were seeded on Lab-Tek ${ }^{\circledR}$ chambered cover glasses (Thermo Scientific ${ }^{\mathrm{TM}}$, Waltham, MA) at a density of $1 \times 10^{4}$ cells $/ \mathrm{cm}^{2}$ and incubated overnight at $37^{\circ} \mathrm{C}, 5 \% \mathrm{CO}_{2}$ and $95 \%$ relative humidity. Then, the cells were treated with $0.2 \mathrm{mg} / \mathrm{mL}$ BODIPY-COOH and BODIPY-CNs in $20 \mu \mathrm{L}$ of transport medium for $4 \mathrm{~h}$. The cells were washed twice with

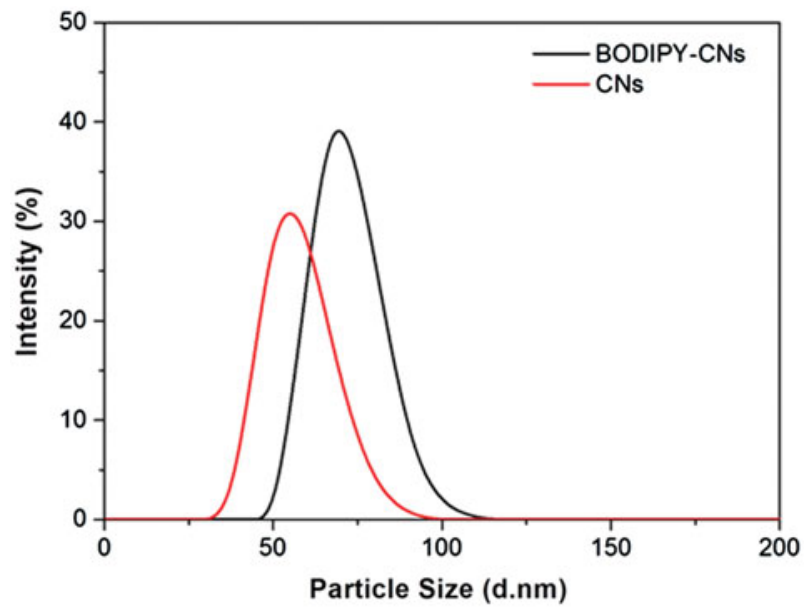

Figure 1. The size distribution by intensity of CNs and BODIPY-CNs.

(A)

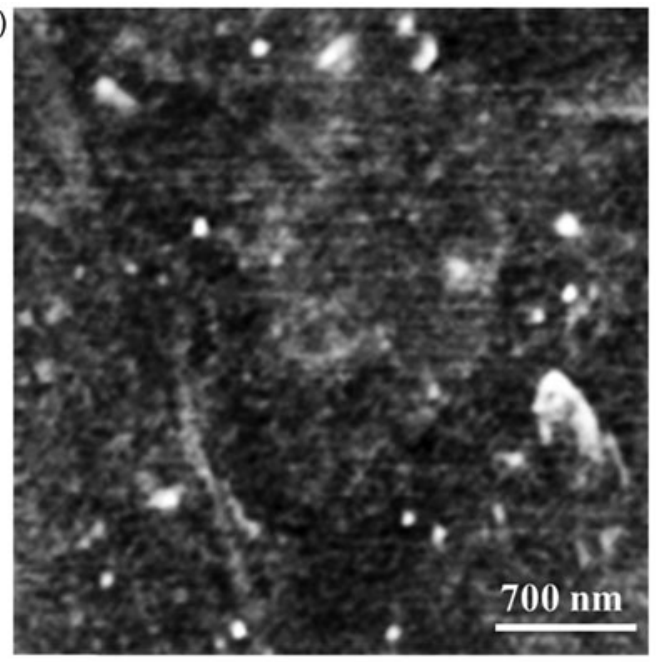

(C)

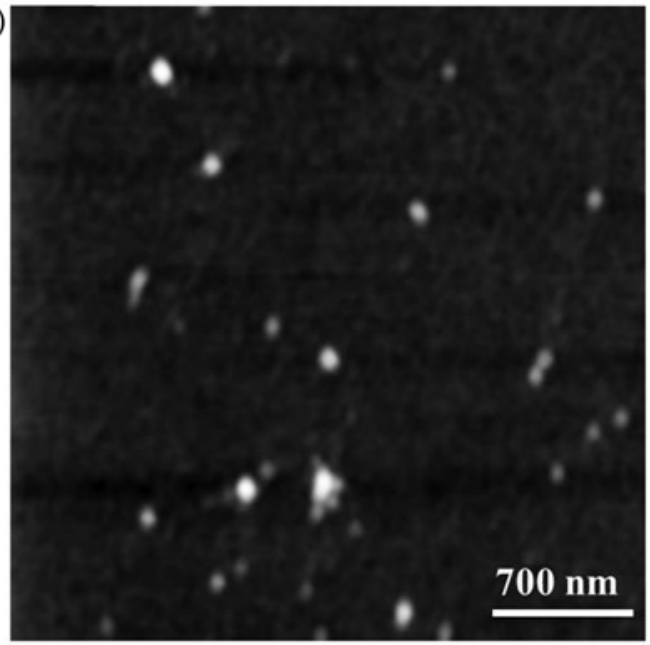

phosphate-buffered saline (PBS), fixed in 3.7\% paraformaldehyde and stained with DAPI (4',6-diamidino-2-phenylindole) before imaging. The applied excitation and emission wavelengths of DAPI dye in this study were 350 and $470 \mathrm{~nm}$, respectively. The excitation and emission wavelengths used here for BODIPY-COOH and BODIPY-CNs were 532 and $640 \mathrm{~nm}$, respectively.

\section{Cell viability assays}

MTT assay was performed to evaluate the cell viability of A549 and BEAS 2B cell lines. Cells were seeded on 96-well micro assay plates at a density of $1 \times 10^{4}$ cells $/ \mathrm{cm}^{2}$ and incubated at $37^{\circ} \mathrm{C}$ in $95 \%$ airs, $5 \% \mathrm{CO}_{2}$ environments for $24 \mathrm{~h}$. BODIPY-CNs were added and incubated for $24 \mathrm{~h}, 48 \mathrm{~h}$ and $72 \mathrm{~h}$. After incubation, the medium was removed and replaced with 3-(4,5-dimethylthiazol-2-yl)-2,5-diphenyltetrazolium bromide (MTT) - containing medium. Plates were incubated for an additional $4 \mathrm{~h}$ at $37^{\circ} \mathrm{C}$. MTT medium was removed and $100 \mu \mathrm{l}$ of DMSO was added to dissolve the formazan crystal. The absorbance was determined using plate reader at a wavelength of $540 \mathrm{~nm}$. Metabolic activity at standard growth conditions was considered 100\%. The concentration inhibiting cell viability by $50 \%$ ( $\mathrm{IC}_{50}$ values) was calculated.
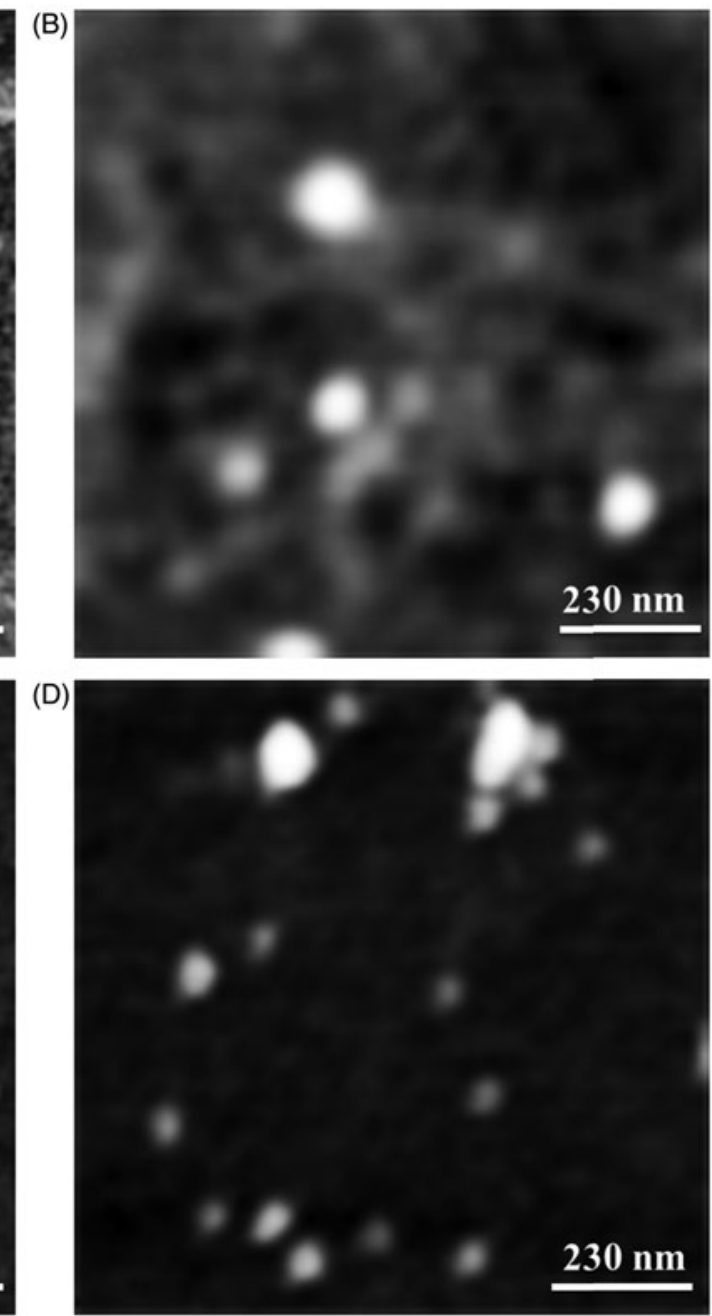

Figure 2. AFM images of (A) CNs at $700 \mathrm{~nm}$, (B) CNs at $230 \mathrm{~nm}$, (C) BODIPY-CNs at $700 \mathrm{~nm}$, (D) BODIPY-CNs at $230 \mathrm{~nm}$ scale. 

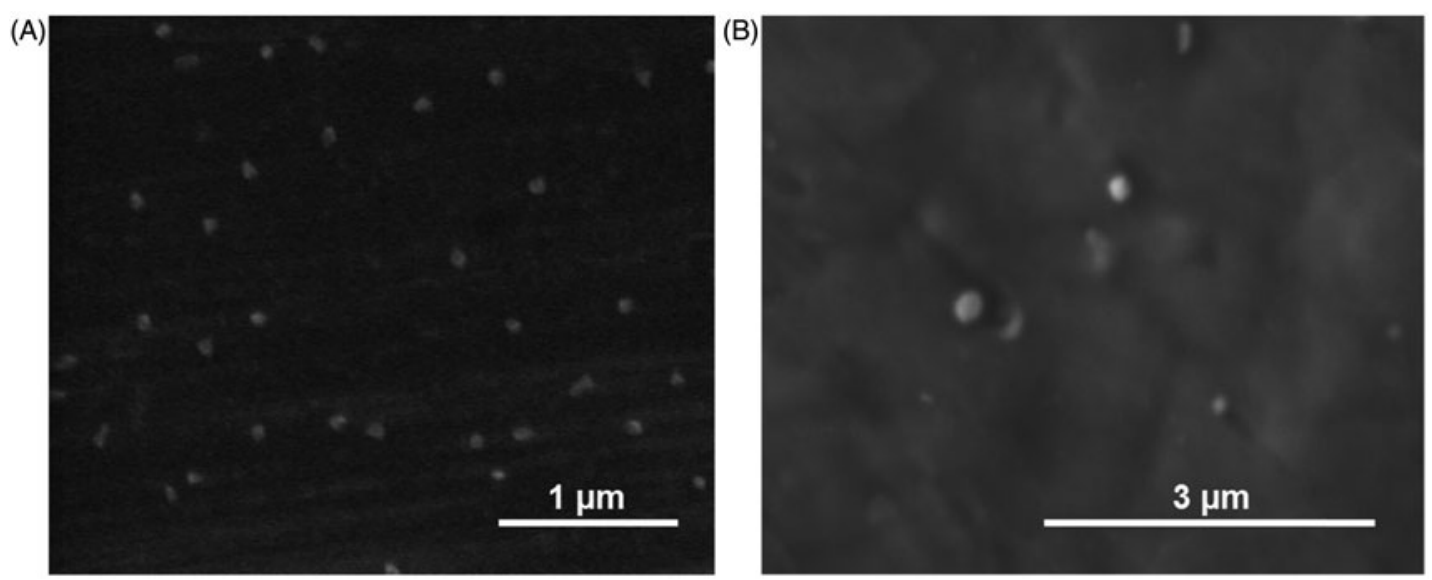

Figure 3. SEM images of (A) CNs at $1 \mu \mathrm{m}$ and (B) BODIPY-CNs at $3 \mu \mathrm{m}$ scale.

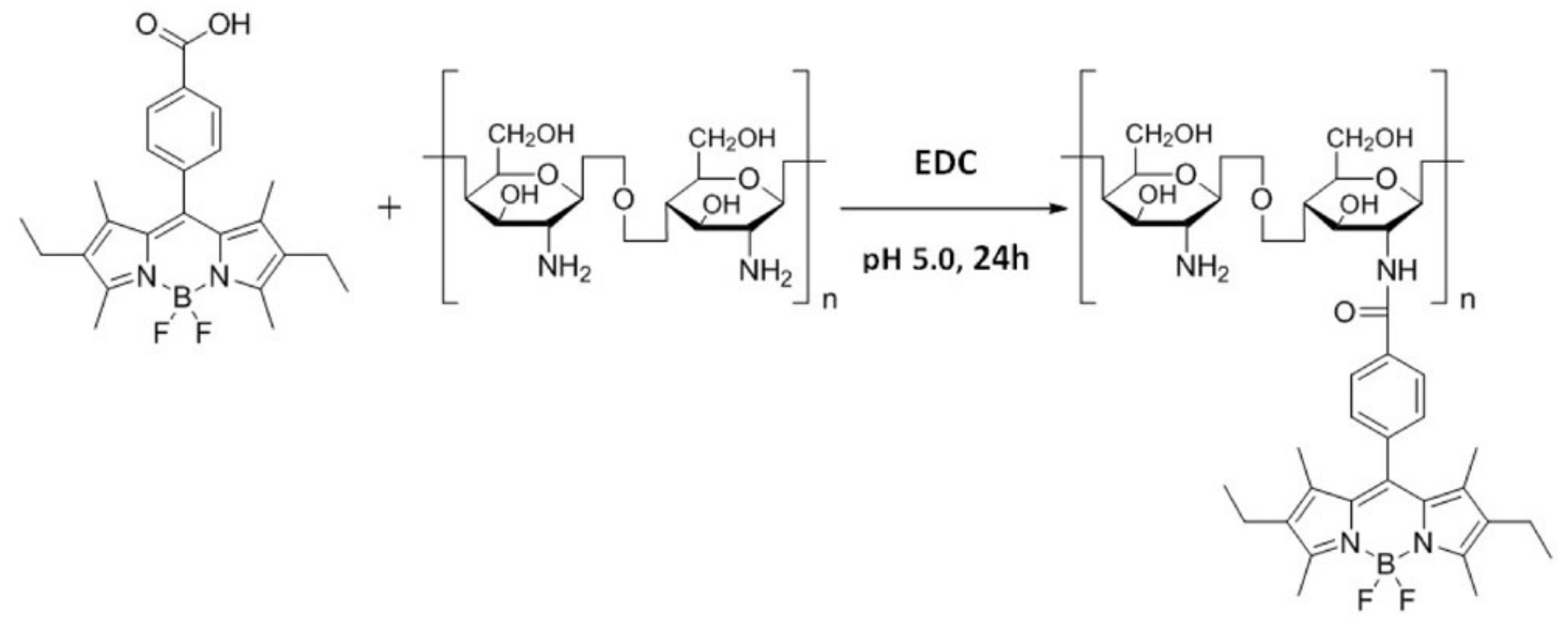

Figure 4. Schematic illustration of the chemical synthesis of BODIPY-COOH conjugated chitosan nanoparticles.

\section{Statistical analysis}

The results represented the mean \pm standard deviation from at least three independent experiments. Statistical significance was assessed by One-way ANOVA using GraphPad Prism5, and with the Dunnett's multiple comparison test.

\section{Results and discussion}

\section{Preparation and characterization of $\mathrm{Ns}$}

Ionic gelation is the convenient method for the fabrication of the chitosan nanoparticles because the process is simple, requires mild conditions and avoids the use of organic solvents and high temperatures (Berger et al., 2004; Nasti et al., 2009). Therefore, in this study, chitosan nanoparticles were synthesized by complexation of polyanionic TPP and cationic chitosan using ionic gelation method at room temperature. The respective average diameters, measured by Zetasizer, of chitosan nanoparticles was about $53.15 \pm 6.47$ as shown in Figure 1, which was consistent with other reports (Bodnár et al., 2005; Calvo et al., 1997). The polydispersity index (PDI) value of chitosan nanoparticles was 0.287 indicating a narrow size distribution with favorable particle size. When chitosan and TPP were mixed with each other, they spontaneously formed compact nano complexes with an overall positive surface charge. The density of the surface charge is reflected by measured zeta $(\zeta)$ potential values, and $\zeta$-potential of prepared CNs were found to be $41.5 \pm 1.8 \mathrm{mV}$ and positive due the presence of the protonated amine groups on the chitosan backbone. The morphology and surface appearance of chitosan nanoparticles were nearly spherical shape, uniform and well dispersed as observed by AFM and SEM (Figure 2(A,B) and Figure 3(A)). The size of chitosan nanoparticles was also obtained via AFM in the range of $58-63 \mathrm{~nm}$. The results were consistent with the measurement of Zetasizer. The ability of the ionic gelation process to form chitosan nanoparticles was assessed by employing FT-IR in order to characterize and compare the functional groups of chitosan and chitosan nanoparticles (data not shown). The changes in functional group of chitosan nanoparticle were consistent with our previous studies (Ilgü et al., 2011). Therefore, we have concluded that the tripolyphosphoric groups of TPP are linked with ammonium groups of chitosan to form nanoparticles. 
Carboxylated BODIPY was synthesized and purified successfully using the method previously described with some modifications (Brizet et al., 2013). The BODIPY$\mathrm{COOH}$ compound was characterized by classical spectroscopic analysis such as NMR spectroscopy and the final product was obtained in 38\% yield.

For synthesis of BODIPY-CNs, the reaction between the chitosan nanoparticles and the BODIPY-COOH was allowed to continue for $24 \mathrm{~h}$ on the magnetic stirrer at room temperature. The presence of a large number of primary amine and hydroxyl groups on the chitosan molecule gave a possibility for many chemical interactions. In acidic $\mathrm{pH}$, amino groups of chitosan can undergo protonation and these reactive groups of $\mathrm{CNs}$ on its polysaccharide chains could make easier coupling reactions with BODIPY-COOH fluorophore. The schematic mechanism of BODIPY-COOH and CNs was illustrated in Figure 4. According to the mechanism, BODIPY-COOH tends to make an interaction with the free ammonium group $\left(-\mathrm{NH}_{2}\right)$ of $\mathrm{CNs}$ due to the randomly coiled

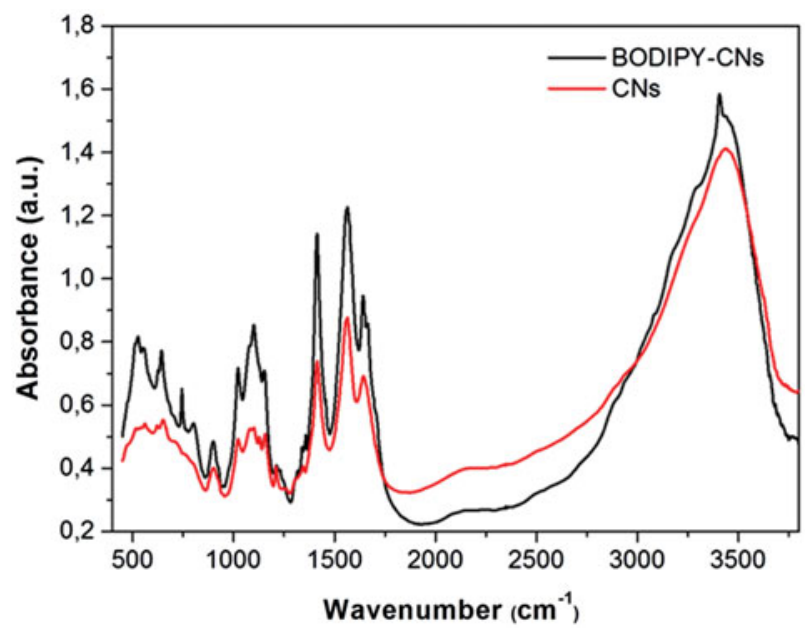

Figure 5. FT-IR spectra of chitosan nanoparticles (CNs) and BODIPY$\mathrm{COOH}$ conjugated chitosan nanoparticles (BODIPY-CNs). arrangement form of particles with TPP at low $\mathrm{pH}$ during ionic gelation process (Sijumon \& Jose, 2010). The BODIPY$\mathrm{COOH}$ amount of the conjugated molecule was measured by UV-visible Spectrophotometry at $510 \mathrm{~nm}$ and the binding efficiency (w/w \%) of BODIPY-COOH to $\mathrm{CNs}$ was calculated as $95 \%$.

In the present study, the complexation of BODIPY-COOH fluorophore and chitosan nanoparticles to form BODIPY-CNs were characterized by physicochemical aspects by several methods. The size distribution of BODIPY-CNs was similar to CNs itself having a narrow batch with the mean size of about $70.25 \pm 11.99 \mathrm{~nm}$. The particle size was slightly larger than chitosan-TPP ones as expected, possibly due to the addition of many BODIPY-COOH fluorophore to CNs.

The zeta potential of BODIPY-CNs nanoparticles reflecting the particle surface charge density decreased to (+) $30.07 \pm 2.6 \mathrm{mV}$ because of reduced protonated amine groups on the chitosan backbone. This simple relationship could be easily explained as coupling reactions of reactive amino groups of $\mathrm{CNs}$ with BODIPY-COOH fluorophore. The interaction of $\mathrm{CNs}$ with BODIPY-COOH fluorophore could also prevent possible aggregation of chitosan nanoparticles and increase their stability. It was previously demonstrated that the strong inter and intramolecular hydrogen bonding of CNs could result in aggregation easily (Fan et al., 2012). The carboxyl groups of the fluorophore may form many possible interactions with $-\mathrm{NH}_{2}$ of $\mathrm{CNs}$ to eliminate nanoparticles from aggregation. The morphology of BODIPY-CNs was visualized by AFM and SEM as shown in Figures 2(C,D) and 3(B). The morphology and surface appearance of BODIPYCNs were similar to chitosan nanoparticles. Both AFM and SEM images for BODIPY-CNs revealed a dense, well dispersed and defined nearly spherical structure. The size of BODIPY-CNs was measured by AFM images in the range of $70-78 \mathrm{~nm}$, which was consistent with the particle size as measured by DLS. The FT-IR studies of BODIPY-CNs were also performed to characterize the changes in the chemical structure of chitosan nanoparticles upon binding to the
(A)

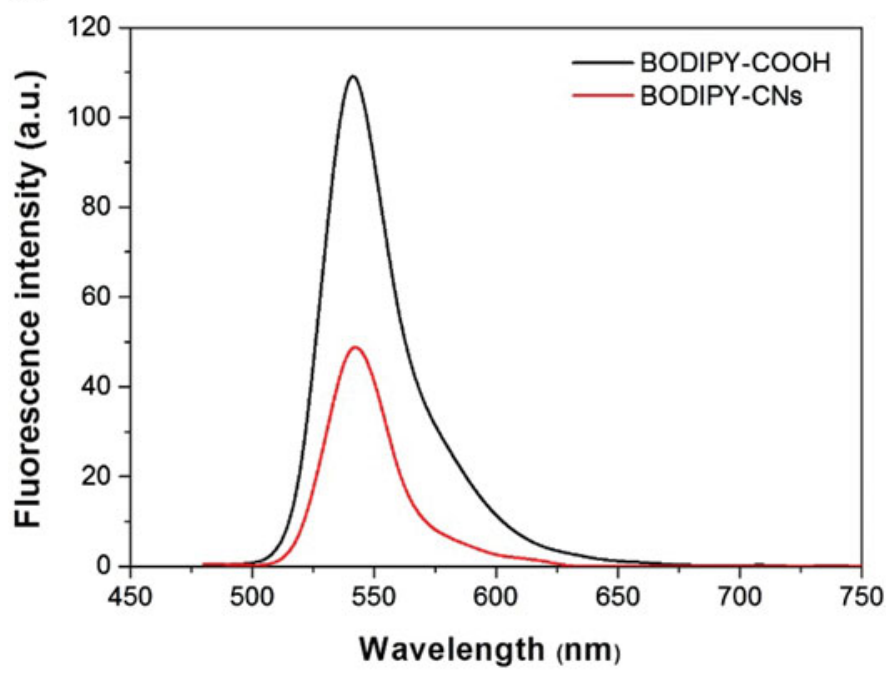

(B)

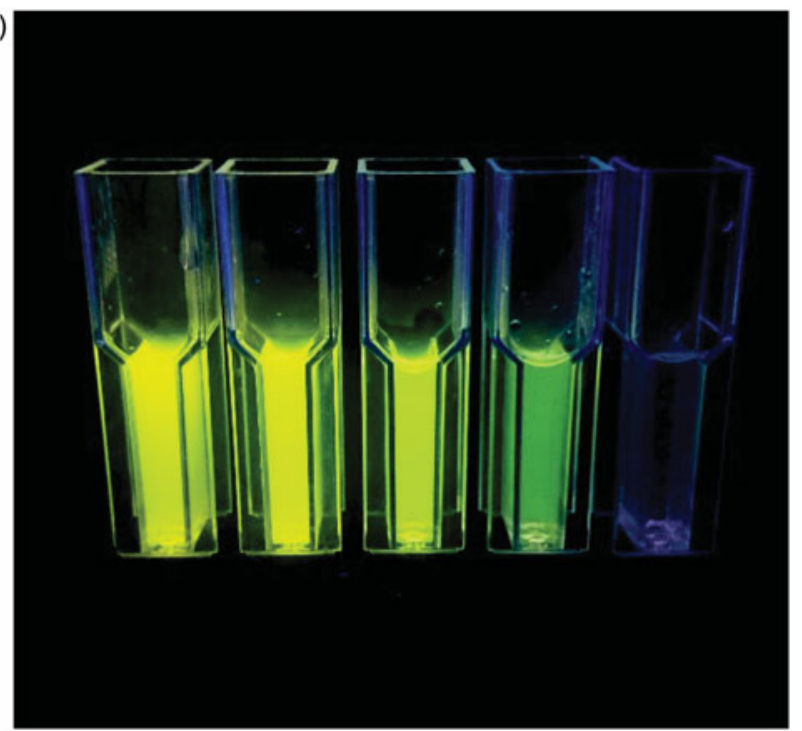

Figure 6. (A) Fluorescence intensities of BODIPY-COOH conjugated chitosan nanoparticles and BODIPY-COOH. (B) Different concentration of BODIPY-CNs $(0.0,0.05,0.1,0.2,0.5 \mathrm{mg} / \mathrm{ml}$ right to left, respectively) under UV light. 

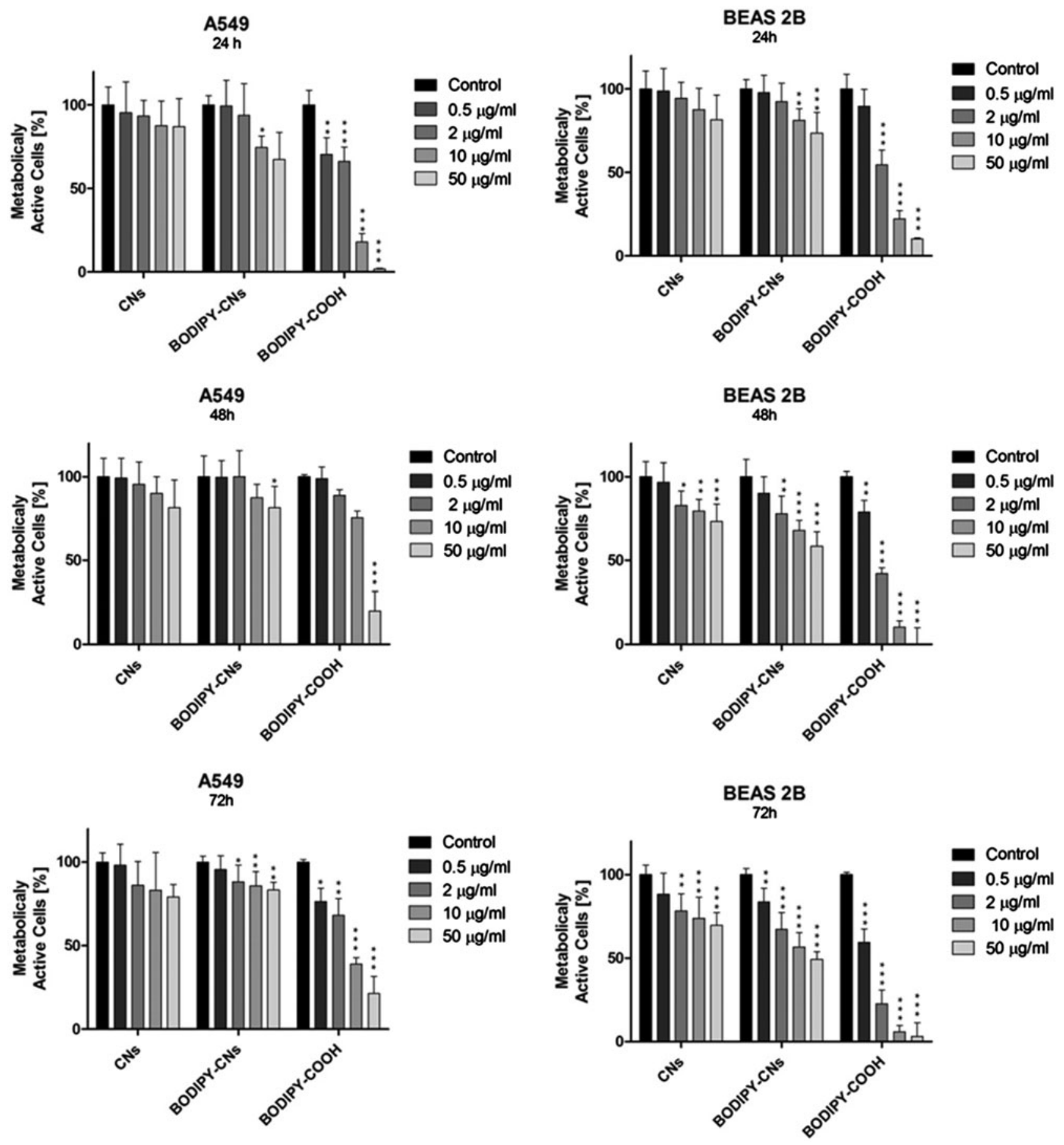

Figure 7. Cell viability of A549 and BEAS 2B cells after the treatment with CNs, BODIPY-CNs and BODIPY-COOH for $24 \mathrm{~h}, 48 \mathrm{~h}$ and $72 \mathrm{~h}$. Each value represents the mean $\pm \mathrm{SD}(n=6)$.

fluorophore (Figure 5). In the spectrum of CNs, a band at $3436 \mathrm{~cm}^{-1}$ indicates the combined peaks of the $-\mathrm{NH}_{2}$ and -OH group stretching vibration. In the spectrum of BODIPYCNs, a shift from $3436 \mathrm{~cm}^{-1}$ to $3440 \mathrm{~cm}^{-1}$ was observed, and the band is getting sharper which indicates the hydrogen bonding enhancement. It has been also demonstrated that the enhanced hydrogen bonding could lead to more compact structure due to bonding of BODIPY-COOH to CNs (de Pinho Neves et al., 2014; Qi et al., 2004). The band of CNs at $1642 \mathrm{~cm}^{-1}$ corresponds to the $\mathrm{CONH}_{2}$ and $-\mathrm{NH}_{2}$ groups. This peak shifted from $1642 \mathrm{~cm}^{-1}$ to $1639 \mathrm{~cm}^{-1}$ in the spectrum of BODIPY-CNs, which shows that the ammonium groups make an interaction with BODIPY-COOH. This finding also confirms the proposed mechanism for the formation of BODIPY-CNs. The peak at $1344 \mathrm{~cm}^{-1}$ shifted to $1339 \mathrm{~cm}^{-1}$ of BODIPY-CNs indicates the existence of $\mathrm{CH}_{3}$ groups. Both CNs and BODIPY-CNs spectrum peaks at $1100 \mathrm{~cm}^{-1}$ are attributed to $\mathrm{C}-\mathrm{O}$ stretching bands.

In the present study, fluorescence properties of BODIPYCNs were determined by fluorescence spectrophotometry (Figure 6A). The results demonstrated that the fluorescent intensity of the chitosan nanoparticles increased due to BODIPY-COOH conjugation. The BODIPY-CNs solution in different concentration exhibited a characteristic green 

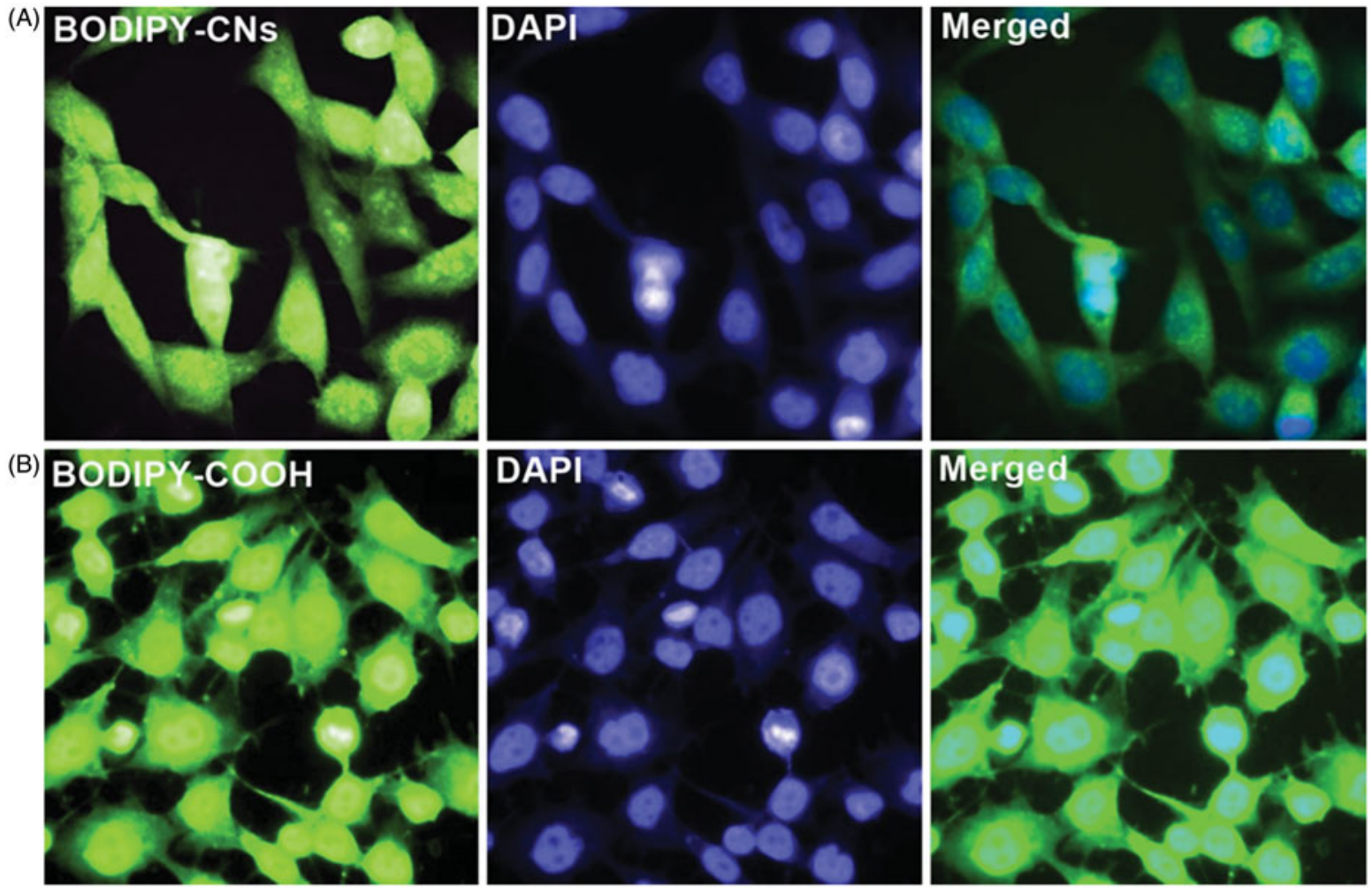

Figure 8. Confocal images of A549 cells treated with $0.2 \mathrm{mg} / \mathrm{mL}$ of (A) BODIPY-CNs, (B) BODIPY-COOH after $4 \mathrm{~h}$ of incubation at $37^{\circ} \mathrm{C}$.

fluorescence of BODIPY-COOH under UV light (Figure 6B). Although $0.1 \mathrm{mg} / \mathrm{ml} \mathrm{CNs}$ was in the first tube, it did not show any fluorescence property, the same amount of BODIPY-CNs reflected a considerable fluorescence intensity. As shown in the third tube, $0.2 \mathrm{mg} / \mathrm{ml}$ BODIPY-CNs displayed a characteristic green fluorescence color.

\section{In vitro cell viability and imaging}

The cytotoxicity of $0.5 \mu \mathrm{g} / \mathrm{mL}, 1 \mu \mathrm{g} / \mathrm{mL}, 2 \mu \mathrm{g} / \mathrm{mL}, 5 \mu \mathrm{g} / \mathrm{mL}$, $10 \mu \mathrm{g} / \mathrm{mL}, 25 \mu \mathrm{g} / \mathrm{mL}$ and $50 \mu \mathrm{g} / \mathrm{mL}$ concentrations of the BODIPY-CNs, CNs and BODIPY-COOH were investigated on A549 cancer cells and BEAS 2B normal cells as control using the MTT assay (Figure 7).

CNs itself did not show any significant toxic effect on both cells types as expected because chitosan is a natural biopolymer and known as biocompatible. Similar results were obtained in other described reports for chitosan and chitosan-related products (de Campos et al., 2004; Huang et al., 2004). On the other hand, when BODIPY-COOH was applied on A549 and BEAS 2B cells, cell viability decreased with the raise in compound concentration as a concentration dependent manner. After $24 \mathrm{~h}$, BODIPY-COOH-treated cells have $\sim 2 \%$ cell viability at $50 \mu \mathrm{g} / \mathrm{ml}$ compound concentration for A549 and 10\% for BEAS 2B cells. The present study demonstrated that application of BODIPY-CNs on both cell lines enhanced the cell viability up to $67 \%$ for A549 and 74\% for BEAS 2B cells. It was also observed that when the incubation time increases up to $72 \mathrm{~h}$, the cytotoxicity of both BODIPY-COOH and BODIPY-CNs was decreased. This finding shows that BODIPY-CNs acts similarly as CNs by lowering cytotoxicity of BODIPY-COOH fluorophore.
Therefore, we can suggest that possible utilization of CNPs together with BODIPY-COOH fluorophore could be a good potential bioimaging agent in various applications in medicine. This new proposed agent would not only act as a fluorescent probe but also be nontoxic for the applied cells.

In order to observe visually the location of BODIPY-CNs in the cells, A549 cancer cells were treated with BODIPYCNs and blue fluorescent dye 4',6-diamidino-2-phenylindole dihydrochloride (DAPI) and then measured by confocal laser scanning microscopy. The present results in Figure 8 show that BODIPY-CNs displayed strong localized fluorescence within the cytoplasm of A549 cells as green color after $4 \mathrm{~h}$ of incubation at $37^{\circ} \mathrm{C}$. For the comparison of visual uptake of BODIPY-COOH fluorophore and BODIPY-CNs in A549 cells, similar intense fluorescence signal were observed along a layer on the cell cytoplasm and there was no apparent difference in the fluorescence signal pattern of two compounds treated cells. The cellular internalization and uptake of both dye changed according to varied concentration of BODIPY-CNs and BODIPY-COOH in a dose-dependent manner (data is not shown). Similar results were reported by other studies showing the uptake of the BODIPY-magnetic nanocomposites increases with dose-dependent manner in A549 and Ishikawa cells (Topel et al., 2015). By treatment of cells with BODIPY-CNs, almost whole cells appeared in good shape. Furthermore, similarly, A549 cells were stained with DAPI for the uptake efficiency and localization profile. DAPI as a marker to the nucleus showed blue fluorescence intensity in the nuclei of A549 cells. The merged image reveals that BODIPY-CNs could specifically bind to A549 cells and localize in their cytoplasm. 


\section{Conclusions}

The present work aims to develop a new fluorescent nanoparticle-based probe for various bioimaging applications that can be used for medical interests. In the development of a multifunctional fluorescent nano-size probe, first, CNs were prepared based on ionic gelation method and BODIPY$\mathrm{COOH}$ fluorescent dye was synthesized as a type of organic fluorescent molecule. Several methods were utilized to determine the physicochemical properties of the $\mathrm{CNs}$ and BODIPY-CNs. Cytotoxic effects of CNs, BODIPY-COOH and BODIPY-CNs were evaluated comparing cell viability of A549 and BEAS 2B cell lines. Confocal images confirmed that the BODIPY-COOH-conjugated chitosan nanoparticles could be utilized efficiently as a multifunctional fluorescent probe. A major advantage of these conjugates is that they are important promising tools for not only in advanced bioimaging systems, but also in intracellular drug delivery, controlled drug release and ultra-sensitive molecular diagnosis events.

\section{Acknowledgements}

The authors would like to thank the Center of Material Research and Biotechnology \& Bioengineering Research Center at Izmir Institute of Technology for their help and technical support.

\section{Declaration of interest}

The authors declare no conflict of interest.

\section{References}

Berger J, Reist M, Mayer JM, et al. (2004). Structure and interactions in covalently and ionically crosslinked chitosan hydrogels for biomedical applications. Eur J Pharm Biopharm 57:19-34.

Bernhard C, Goze C, Rousselin Y, Denat F. (2010). First bodipy-DOTA derivatives as probes for bimodal imaging. Chem Commun (Camb) 46:8267-8269.

Bodnár M, Hartmann JF, Borbély J. (2005). Nanoparticles from Chitosan. Macromol Symp 227:321-326.

Boens N, Leen V, Dehaen W. (2012). Fluorescent indicators based on BODIPY. Chem Soc Rev 41:1130-1172.

Brizet B, Bernhard C, Volkova Y, et al. (2013). Boron functionalization of BODIPY by various alcohols and phenols. Org Biomol Chem 11: 7729-7737.

Calvo P, Remuñán-López C, Vila-Jato JL, Alonso MJ. (1997). Novel hydrophilic chitosan-polyethylene oxide nanoparticles as protein carriers. J Appl Polym Sci 63:125-132.

Chan J, Dodani SC, Chang CJ. (2012). Reaction-based small-molecule fluorescent probes for chemoselective bioimaging. Nat Chem 4:973-984.
Chekina N, Horak D, Jendelova P, et al. (2011). Fluorescent magnetic nanoparticles for biomedical applications. J Mater Chem 21: 7630-7639.

Corr SA, Rakovich YP, Gun'ko YK. (2008). Multifunctional magneticfluorescent nanocomposites for biomedical applications. Nanoscale Res Lett 3:87-104.

de Campos AM, Diebold Y, Carvalho EL, et al. (2004). Chitosan nanoparticles as new ocular drug delivery systems: in vitro stability, in vivo fate, and cellular toxicity. Pharm Res 21:803-810.

de Pinho Neves AL, Milioli CC, Müller L, et al. (2014). Factorial design as tool in chitosan nanoparticles development by ionic gelation technique. Colloids Surf A Physicochem Eng Asp 445:34-39.

Fan W, Yan W, Xu Z, Ni H. (2012). Erythrocytes load of low molecular weight chitosan nanoparticles as a potential vascular drug delivery system. Colloids Surf B Biointerfaces 95:258-265.

Huang M, Khor E, Lim L-Y. (2004). Uptake and cytotoxicity of chitosan molecules and nanoparticles: effects of molecular weight and degree of deacetylation. Pharm Res 21:344-353.

İlgü H, Turan T, Şanli-Mohamed G. (2011). Preparation, characterization and optimization of chitosan nanoparticles as carrier for immobilization of thermophilic recombinant esterase. J Macromol Sci Part A 48: 713-721.

Jayakumar R, Menon D, Manzoor K, et al. (2010). Biomedical applications of chitin and chitosan based nanomaterials-A short review. Carbohydr Polym 82:227-232.

Kobayashi H, Ogawa M, Alford R, et al. (2010). New strategies for fluorescent probe design in medical diagnostic imaging. Chem Rev 110:2620-2640.

Kumar MNVR, Muzzarelli RAA, Muzzarelli C, et al. (2004). Chitosan chemistry and pharmaceutical perspectives. Chem Rev 104: 6017-6084.

Loudet A, Burgess K. (2007). BODIPY dyes and their derivatives: syntheses and spectroscopic properties. Chem Rev 107:4891-4932.

Nasti A, Zaki NM, de Leonardis P, et al. (2009). Chitosan/TPP and chitosan/TPP-hyaluronic acid nanoparticles: systematic optimization of the preparative process and preliminary biological evaluation. Pharm Res 26:1918-1930.

Nune SK, Gunda P, Thallapally PK, et al. (2009). Nanoparticles for biomedical imaging. Expert Opin Drug Deliv 6:1175-1194.

Qi L, Xu Z, Jiang X, Hu C, Zou X. (2004). Preparation and antibacterial activity of chitosan nanoparticles. Carbohydr Res 339:2693-2700.

Rinaudo M. (2006). Chitin and chitosan: properties and applications. Prog Polym Sci 31:603-632.

Sijumon K, Jose S. (2010). Understanding the mechanism of ionic gelation for synthesis of chitosan nanoparticles using qualitative techniques. Asian J Pharmaceut 4:148-153.

Terai T, Nagano T. (2008). Fluorescent probes for bioimaging applications. Curr Opin Chem Biol 12:515-521.

Topel SD, Topel O, Bostancioglu RB, Koparal AT. (2015). Synthesis and characterization of Bodipy functionalized magnetic ironoxide nanoparticles for potential bioimaging applications. Colloids Surf B: Biointerfaces 128:245-253.

Ulrich G, Ziessel R, Harriman A. (2008). The chemistry of fluorescent bodipy dyes: versatility unsurpassed. Angew Chem Int Ed Engl 47: 1184-1201.

Yuan L, Lin W, Zheng K, Zhu S. (2013). FRET-based small-molecule fluorescent probes: rational design and bioimaging applications. Acc Chem Res 46:1462-1473.

Zhao J, Wu J. (2006). Preparation and characterization of the fluorescent chitosan nanoparticle probe. Chin J Anal Chem 34:1555-1559. 